DOI: 10.12957/demetra.2017.24862

\title{
Análise nutricional quantitativa de uma dieta da proteína destinada a todos os públicos
}

\section{Quantitative nutritional analysis of a protein diet aimed at all audiences}

\author{
Luciana Neves Faria' \\ Anelise Andrade de Souza ${ }^{1}$ \\ 1 Universidade Federal de Ouro Preto, Escola \\ de Nutrição, Departamento de Alimentos. Ouro \\ Preto-MG, Brasil. \\ Correspondência / Correspondence \\ Anelise Andrade de Souza \\ Universidade Federal de Ouro Preto, Escola de \\ Nutrição, s/n. Campus Universitário, Morro do \\ Cruzeiro, 35400-000 - Ouro Preto-MG, Brasil. \\ E-mail asouzandrade@yahoo.com.br
}

\section{Resumo}

As dietas da moda podem ser definidas como padrões de comportamento alimentar não usuais e temporários, adotados por seguidores de forma entusiástica, promovendo resultados rápidos e atraentes, mas sem possuir nenhum fundamento científico. Entre elas, podem-se citar as dietas da proteína. O presente estudo teve como objetivo realizar análise nutricional quantitativa de um cardápio semanal de uma dieta da proteína publicada em um livro impresso e também disponibilizada em mídia digital, destinada a todos os públicos. Para a realização deste estudo, foi construído um modelo feminino e um masculino em sobrepeso, ambos com possibilidade de serem eventuais utilizadores da dieta. Os cardápios avaliados do livro referem-se à fase de indução da dieta da proteína. Foram realizadas análises quantitativas de energia e dos nutrientes: carboidratos, proteínas, lipídeos, fibras, colesterol, cálcio, ferro e vitamina C. Os resultados do estudo indicaram um planejamento alimentar inadequado para os modelos em sobrepeso construídos, apresentando uma dieta hiperproteica, hiperlipídica, hipoglicídica e hipercalórica, além de valores acima do recomendado para o nutriente colesterol e abaixo do recomendado para os nutrientes cálcio e fibras. Concluiu-se que a dieta da proteína não pode ser considerada saudável e equilibrada, não atendendo às necessidades nutricionais individuais, sendo importante a realização de campanhas de educação nutricional para a população, esclarecendo os malefícios que uma dieta desbalanceada e sem acompanhamento nutricional pode gerar ao estado nutricional.

Palavras-chave: Dietas da moda. Emagrecimento. Sobrepeso. Planejamento Alimentar. 


\section{Abstract}

Fad diets can be defined as unusual eating behavior patterns, popular and temporary, adopted by followers enthusiastically promoting quick and attractive results, but without having any scientific basis. Among the fad diets can be mentioned protein diets. Thus, this study aimed to carry out quantitative nutritional analysis of a weekly menu of a diet of protein published in a printed book and also available in digital media, aimed at all audiences. For the study was built a female model and a male overweight, both with possibility of potential users of the diet. The menus reviews of the book used in this study were constructed from the same orientations and relate to the induction phase of protein diet. Quantitative analysis of energy and the following macronutrients and micronutrients were performed: carbohydrates, proteins, lipids, fiber, cholesterol, calcium, iron and vitamin C. This study found inadequate food planning for models built in overweight. The results show a high protein, high fat, low-carbohydrate and high calorie diet, and above the recommended values for the nutrient cholesterol and lower than recommended for the nutrients calcium and fiber. Concluded that the protein diet is far from being healthy and balanced, since it does not consider the individual nutritional needs. It is therefore of utmost importance that nutrition education campaigns are made to the general population, so that is clear about the harm that an unbalanced diet and no nutritional monitoring can lead to nutritional status.

Keywords: Fad Diets. Loss Weight. Overweight. Meal Planning.

\section{Introdução}

Nos dias de hoje, a alimentação é definida pelo estilo de vida moderno, marcado pela falta de tempo para preparo e consumo de alimentos, levando à emergência de preparações do tipo fast food. ${ }^{1}$ E como consequência desse tipo de alimentação, observa-se um aumento do consumo de ácidos graxos saturados, açúcares, refrigerantes, álcool, produtos industrializados com excesso de ácidos graxos "trans" e, ao mesmo tempo, uma redução considerável no consumo de carboidratos complexos, frutas, verduras e legumes. ${ }^{2}$

Esse fato gera um quadro de excesso calórico por conta da elevada ingestão de macronutrientes e deficiência de micronutrientes, além das fibras, ${ }^{2}$ que, aliado à redução da atividade física, associase à alta prevalência de Doenças Crônicas Não Transmissíveis (DCNT), como a obesidade, e à diminuição da qualidade de vida da população. ${ }^{3}$ 
Com o aumento da prevalência da obesidade no Brasil e devido a todos os problemas ocasionados pela doença, em conjunto com a atual imposição da sociedade, que considera normal o corpo magro e/ou musculoso, a população passa a entender o biotipo magro como sinal de saúde, beleza e qualidade de vida e, dessa maneira, busca formas de possuir um corpo esbelto, que nunca esteve tão em evidência como nos tempos atuais. ${ }^{4}$

Assim, regularmente são lançadas, de modo impresso ou on-line, as denominadas "dietas da moda", induzindo a população a ilusões de emagrecimento rápido e sem sacrifício, que, além de não estimularem a mudança de hábitos alimentares e a necessidade da prática de atividade física, não propiciam hábitos saudáveis e são inadequadas do ponto de vista nutricional, o que, em longo prazo, pode comprometer a saúde em muitos aspectos. ${ }^{4}$

A mídia digital e impressa vem contribuindo, sobremaneira, para o surgimento de conceitos errôneos a respeito de saúde na busca de um "corpo ideal”, veiculando várias "dietas da moda", além de dicas, simpatias, depoimentos pessoais, tabelas de calorias e outras informações não científicas com o objetivo de perda de peso. ${ }^{4,5}$

Neste estudo, buscou-se avaliar quantitativamente uma "dieta da moda" denominada "dieta da proteína" veiculada em mídia impressa e digital, destinada a todos os públicos, e a partir dos resultados encontrados, orientar a população e profissionais de saúde sobre os possíveis riscos da utilização desta dieta.

\section{Metodologia}

O estudo é de corte descritivo e para sua realização foi adquirido um livro específico sobre "dieta da proteína", ${ }^{6}$ publicado por editora, e também disponibilizado em mídia digital.

A partir das informações do livro, foi construído um cardápio semanal analisado por meio do software Virtual Nutri Plus ${ }^{\circledR}$, versão banco de dados. ${ }^{7}$ Quando alguns alimentos ou preparações não estavam presentes no banco de dados do software, adquiria-se a informação da composição nutricional por meio de rótulos de alimentos ou preparações similares.

Foram realizadas análises quantitativas de energia e dos seguintes nutrientes: carboidratos, proteínas, lipídeos, fibras, colesterol, cálcio, ferro e vitamina C dos cardápios presentes no livro. A média dos resultados foi obtida com ajuda do software Microsoft Office Excel ${ }^{\circledR}$ versão $2010 .{ }^{8}$

A distribuição percentual de macronutrientes encontrados no estudo foi comparada com as recomendações da Food and Agriculture Organization, que orienta: carboidratos, $55 \%$ a 75\%; proteína, $10 \%$ a $15 \%$ e lipídios, $15 \%$ a $30 \% .^{9}$ 
Já os valores encontrados das fibras e dos micronutrientes cálcio, ferro, colesterol e vitamina $\mathrm{C}$ foram comparados com a Dietary Reference Intakes (DRIs). ${ }^{10,11}$ Inicialmente, utilizou-se a Recommended Dietary Allowances (RDA), porém, quando esses valores não estavam disponíveis nas DRIs, a comparação foi realizada com o Adequate Intake (AI). ${ }^{12}$ A ocorrência de indicação em excesso do consumo de alguns micronutrientes na dieta estudada foi verificada com o auxílio da Tolerable Upper Intake Level (UL). ${ }^{12}$

Para verificar a adequação do cardápio semanal avaliado às recomendações nutricionais e percentuais de distribuição dos nutrientes, fez-se necessário a construção de modelos feminino e masculino em sobrepeso, possíveis usuários da “dieta da proteína” (Quadro 1).

Quadro 1. Caracterização dos modelos 1 e 2.

\begin{tabular}{|c|c|c|}
\hline Características dos modelos & Modelo 1 & Modelo 2 \\
\hline Sexo & Feminino & Masculino \\
\hline Idade & 43 anos & 57 anos \\
\hline Peso $(\mathrm{kg})$ & 84 & 109 \\
\hline Altura $(\mathrm{m})$ & 1,65 & 1,75 \\
\hline IMC $\left(\mathrm{kg} / \mathrm{m}^{2}\right)$ & 30,85 & 35,59 \\
\hline Fator Atividade & Leve & Leve \\
\hline
\end{tabular}

Para caracterização dos modelos feminino e masculino em sobrepeso, foi utilizado o Índice de Massa Corporal (IMC), que, embora possua limitações em seu uso, é altamente aplicado na prática clínica. Para o cálculo do Gasto Energético Total (GET) dos dois modelos, recorreu-se à fórmula em que se determina a Taxa do Metabolismo Basal (TMB) e fatores atividade (FA) relacionados a um indivíduo com atividade leve. ${ }^{13}$

Com base nas informações do modelo 1, o IMC obtido foi de $30,86 \mathrm{~kg} / \mathrm{m}^{2}$, sendo classificado com sobrepeso II, e para o modelo 2, o IMC obtido foi de $35,59 \mathrm{~kg} / \mathrm{m}^{2}$, sendo também classificado com sobrepeso II.

O método recomendado para a perda de peso envolve um planejamento dietético correto que promova uma restrição calórica leve, com a taxa de perda de peso não devendo ultrapassar 1 quilo por semana. ${ }^{14}$ Considerando que a perda ponderal proposta de ambos os modelos em um mês será de 2 quilos (0,5 quilo por semana), a dieta foi estimada em um valor de 1919,96 Kcal/ dia para o modelo 1 e $2.808,94 \mathrm{kcal} /$ dia para o modelo 2. 
As recomendações de fibras e dos micronutrientes cálcio, colesterol, ferro e vitamina C utilizadas para a comparação dos valores encontrados na avaliação da dieta da proteína com a necessidade dos modelos 1 e 2 estão listadas na Tabela 1 e seguem os valores preconizados pelo Dietary Reference Intakes (DRI) ${ }^{10,11}$ (Tabela 1).

Tabela 1. Ingestão diária recomendada dos nutrientes: cálcio, colesterol, ferro, fibras e vitamina C, Brasil, 2016.

\begin{tabular}{ccccc}
\hline & \multicolumn{2}{c}{ Modelo 1 } & \multicolumn{2}{c}{ Modelo 2 } \\
\hline Micronutrientes & RDA OU AI & UL & RDA OU AI & UL \\
Cálcio $(\mathrm{mg})$ & $1000^{* *}$ & 2500 & $1200^{* *}$ & 2500 \\
Colesterol $(\mathrm{mg})$ & $<200$ & - & $<200$ & - \\
Ferro $(\mathrm{mg})$ & $18^{*}$ & 45 & $8 *$ & - \\
Fibras $(\mathrm{g})$ & $25^{* *}$ & - & $30 * *$ & 2000 \\
\hline Vitamina C $(\mathrm{mg})$ & $75^{*}$ & 2000 & $90^{*}$ & \\
\hline
\end{tabular}

* Valores de $\mathrm{RDA}^{12}$; **Valores de $\mathrm{AI}^{12}$.

\section{Resultados e Discussão}

A Tabela 2 mostra as médias semanais energéticas dos macronutrientes carboidrato, proteína e lipídeo, calculadas a partir da análise dos cardápios produzidos com base nas orientações do livro da "dieta da proteína" e a adequação dos valores médios encontrados em relação às necessidades nutricionais dos modelos 1 e 2. 
Tabela 2. Médias semanais de energia da fase da dieta de indução e comparação com as necessidades nutricionais dos modelos 1 e 2, Brasil, 2016.

\begin{tabular}{|c|c|c|c|c|}
\hline & $\begin{array}{c}\text { Energia } \\
\text { (kcal) }\end{array}$ & $\begin{array}{c}\text { Carboidrato } \\
(\mathrm{g})\end{array}$ & Proteína (g) & Lipídeo (g) \\
\hline $\begin{array}{c}\text { Média semanal dos } \\
\text { cardápios }\end{array}$ & 2531,93 & 20 & 136,44 & 168,40 \\
\hline$\%$ Macronutrientes & - & 4,16 & 28,43 & 78,94 \\
\hline $\begin{array}{c}\text { Necessidades } \\
\text { nutricionais modelo } 1\end{array}$ & 1919,96 & $55-75 \% *$ & $10-15 \% *$ & $15-30 \% *$ \\
\hline \multirow[t]{2}{*}{ Adequação (\%) } & 131,87 & 6,93 & 189,53 & 315,76 \\
\hline & $\begin{array}{c}\text { Energia } \\
\text { (kcal) }\end{array}$ & $\begin{array}{c}\text { Carboidrato } \\
(\mathrm{g})\end{array}$ & Proteína (g) & Lipídeo (g) \\
\hline $\begin{array}{c}\text { Média semanal dos } \\
\text { cardápios }\end{array}$ & 3958,75 & 20 & 211,56 & 262,85 \\
\hline$\%$ Macronutrientes & - & 2,85 & 30,13 & 84,22 \\
\hline $\begin{array}{c}\text { Necessidades } \\
\text { nutricionais modelo } 2\end{array}$ & 2808,94 & $55-75 \% *$ & $10-15 \% *$ & $15-30 \% *$ \\
\hline Adequação (\%) & 140,9 & 4,8 & 200,9 & 336,9 \\
\hline
\end{tabular}

Fonte: *9.

Em relação aos resultados encontrados na avaliação dos valores energéticos e na distribuição dos macronutrientes, observaram-se planejamentos hipercalóricos, hiperproteicos, hiperlipídicos e hipoglicídicos, quando comparado às necessidades nutricionais dos dois modelos criados.

A perda de peso inicial, que acontece com a grande maioria dos pacientes submetidos a dietas da proteína, com essas características citadas no parágrafo anterior, ocorre devido ao esvaziamento das reservas de glicogênio e água. Como consequência, a perda de peso poderia ser, preponderantemente, uma perda de fluidos, levando à desidratação do paciente. ${ }^{15}$ Além desse fator, a redução da secreção da insulina, devido à nulidade dos carboidratos nas refeições, promove a mobilização de ácidos graxos e corpos cetônicos, aumentando ainda mais o catabolismo proteico. O paciente perde peso por causa da perda de massa magra. ${ }^{16}$ Ao final de 12 meses de dieta, estudiosos observaram que pacientes submetidos à dieta da proteína tiveram o mesmo resultado ou resultados 
inferiores aos pacientes submetidos a planejamentos alimentares adequados, com distribuição correta dos nutrientes, isso porque a regulação da saciedade, ocorrida na dieta da proteína, tem seu efeito limitado pela ação de dois hormônios, a insulina e a leptina, que possuem efeito estimulante sobre a ingestão alimentar, aumentando, consequentemente, a adiposidade. Gorduras também são menos eficientes que proteínas e carboidratos no envio dos sinais de saciedade, fazendo com que o paciente em dieta da proteína coma mais e em quantidades maiores. ${ }^{17}$

Outro fator negativo da utilização da dieta da proteína diz respeito às mudanças metabólicas ocorridas, com aumento da resistência à insulina e, por conseguinte, intolerância à glicose e hiperinsulinemia, que são similares àquelas percebidas na obesidade hipotalâmica ou de origem genética e é considerada a primeira manifestação clínica do diabetes mellitus tipo 2.18 Ademais, dieta hiperlipídica também é um dos principais fatores que auxiliam no aumento dos níveis de lipídios sanguíneos e, dessa forma, a origem dos acidentes cardiovasculares. A incidência de doenças cardiovasculares e outras doenças vasculares estão congregadas aos distúrbios ocorridos no metabolismo lipídico, resultando nas dislipidemias, com altas concentrações séricas de colesterol e de triglicerídeos. ${ }^{17}$

Estudos sugerem também precaução com dietas hiperproteicas em indivíduos com evidências de doença renal, diabéticos e com histórico de nefrolitíase, devido a esta dieta estar associada a uma maior excreção urinária de cálcio em indivíduos saudáveis, e hipercalciúria, sendo uma consequência do aumento da síntese de ácido durante a oxidação de aminoácidos sulfurados, encontrados, especialmente, em proteínas de origem animal. ${ }^{19}$ Também nestas dietas hiperproteicas e hipoglicídicas, por conta da limitação de carboidratos, ocorre a diminuição de peso pela perda de fluidos, resultando em desidratação..$^{20}$ Mesmo que as proteínas sejam nutrientes vitais para a manutenção de tecidos e para o metabolismo, o excesso pode causar agravos à saúde, como: aterosclerose, câncer, doenças renais e osteoporose. Tais fatores interferem na liberação renal de ácido úrico e, consequentemente, elevam os níveis séricos desse ácido, o que pode levar ao aparecimento da Gota. ${ }^{4}$

Da mesma forma, as dietas da moda consideradas de baixo valor energético irão promover a redução do metabolismo basal pela perda de massa muscular, utilizada para fornecer energia ao organismo, o que pode tornar ainda mais difícil o processo de perda de peso e sua manutenção em longo prazo. Estudos apontam que dietas hipoenergéticas, independentemente do tipo de proposta, não são eficazes para perda de peso em longo prazo. Além disso, cerca de $80 \%$ dos pacientes recuperam o peso perdido após um mês da finalização da dieta e o abandono do tratamento é de, aproximadamente, $50 \% .{ }^{20}$ Para a ingestão energética correta, deve-se considerar a TMB individual como valor mínimo de ingestão calórica, não podendo haver planejamento alimentar baseado em dietas abaixo desta taxa, a fim de evitar riscos à saúde., ${ }^{4,21}$ 
Dietas muito restritivas, hipocalóricas ou hipercalóricas inadequadas em relação à distribuição dos macronutrientes, caracterizadas pela pouca variedade de alimentos permitidos, como a dieta avaliada neste estudo, podem acarretar carências nutricionais específicas.

A Tabela 3 mostra as médias semanais dos nutrientes cálcio, colesterol, ferro e vitamina C, além das fibras, calculadas também a partir da análise dos cardápios da "dieta da proteína”, e a adequação dos valores médios encontrados em relação às necessidades nutricionais dos modelos 1 e 2 (Tabela 3).

Tabela 3. Médias semanais dos nutrientes da fase da dieta de indução e comparação com as necessidades nutricionais dos modelos 1 e 2, Brasil, 2016.

\begin{tabular}{|c|c|c|c|c|c|c|c|}
\hline & Cálcio (mg) & $\begin{array}{l}\text { Colesterol } \\
(\mathbf{m g})\end{array}$ & \multicolumn{2}{|c|}{ Ferro (mg) } & Fibras (g) & $\begin{array}{r}\text { Vitar } \\
(\mathbf{r}\end{array}$ & lina $C$ \\
\hline $\begin{array}{c}\text { Média semanal dos } \\
\text { cardápios }\end{array}$ & 925,4 & 1687,3 & \multicolumn{2}{|c|}{18,2} & 6,3 & & 1,6 \\
\hline \multirow{2}{*}{$\begin{array}{c}\text { Necessidades } \\
\text { nutricionais } \\
\text { modelo } 1\end{array}$} & $\begin{array}{c}\text { RDA } \\
\text { ou AI }\end{array}$ & RDA ou AI & $\begin{array}{l}\text { RDA } \\
\text { ou AI }\end{array}$ & UL & RDA ou AI & $\begin{array}{l}\text { RDA } \\
\text { ou AI }\end{array}$ & $\mathrm{UL}$ \\
\hline & $1000^{* *} 2500^{* * *}$ & $<200$ ** & $18^{* *}$ & $45^{* *}$ & $25^{* *}$ & $75^{* *}$ & $2000 * *$ \\
\hline \multirow[t]{2}{*}{ Adequação (\%) } & 92,5 & 843,7 & \multicolumn{2}{|c|}{101,1} & 25,3 & \multicolumn{2}{|c|}{148,8} \\
\hline & Cálcio (mg) & $\begin{array}{l}\text { Colesterol } \\
\quad(\mathrm{mg})\end{array}$ & \multicolumn{2}{|c|}{ Ferro (mg) } & Fibras (g) & \multicolumn{2}{|c|}{$\begin{array}{c}\text { Vitamina C } \\
(\mathbf{m g})\end{array}$} \\
\hline $\begin{array}{c}\text { Média semanal dos } \\
\text { cardápios }\end{array}$ & 1037,8 & 2561,9 & \multicolumn{2}{|c|}{23,5} & 6,4 & \multicolumn{2}{|c|}{131,5} \\
\hline \multirow{2}{*}{$\begin{array}{c}\text { Necessidades } \\
\text { nutricionais } \\
\text { modelo } 2\end{array}$} & $\begin{array}{c}\text { RDA UL } \\
\text { ou AI }\end{array}$ & RDA ou AI & $\begin{array}{l}\text { RDA } \\
\text { ou AI }\end{array}$ & $\mathrm{UL}$ & RDA ou AI & $\begin{array}{l}\text { RDA } \\
\text { ou AI }\end{array}$ & $\mathrm{UL}$ \\
\hline & $1200^{* *} 2500^{* *}$ & $<200^{* *}$ & $8 * *$ & $45^{* *}$ & $30 * *$ & $90 * *$ & $2000^{* *}$ \\
\hline Adequação (\%) & 86,48 & 1280,9 & \multicolumn{2}{|c|}{293,75} & 21,3 & \multicolumn{2}{|c|}{146,1} \\
\hline
\end{tabular}

Fonte: *9;**12. 
Em relação ao consumo de micronutrientes como o cálcio, ferro e vitamina C, assim como as fibras, os mesmos são fundamentais e indispensáveis para um desenvolvimento adequado e manutenção da homeostase no organismo, e por isso precisam estar presentes no planejamento alimentar em quantidades corretas. ${ }^{22-26}$ No estudo em questão, o ferro e vitamina C se apresentaram em quantidades médias adequadas no cardápio produzido para uma semana de atendimento, ao contrário dos nutrientes, cálcio, colesterol e fibras.

O ferro está envolvido em diversas reações do organismo, como o transporte de oxigênio no sangue, o armazenamento de oxigênio no tecido muscular, síntese de DNA e metabolismo energético, além de participar na defesa do organismo. Sua deficiência causa consequências graves, sendo a anemia a patologia mais frequente, acarretando implicações como fraqueza, cansaço, perda de concentração, palpitação, dor de cabeça, desânimo, palidez, sonolência, diminuição da capacidade respiratória, podendo levar à morte. ${ }^{22} \mathrm{O}$ excesso de ferro também pode causar danos ao organismo devido aos seus efeitos tóxicos, podendo se refletir em dores abdominais, perda de peso, perda de memória, quedas de cabelo, atrofia testicular, cirrose hepática e até diabetes. ${ }^{22}$

A vitamina C é uma vitamina hidrossolúvel e termolábil. Os seres humanos e outros primatas são os únicos mamíferos incapazes de sintetizá-la. É de suma importância para diversos fatores do organismo, como a prevenção do escorbuto, defesa do organismo contra infecções, formação das fibras colágenas existentes em praticamente todos os tecidos do corpo humano, como derme, cartilagem e ossos, além de ser fundamental na integridade das paredes dos vasos sanguíneos. Sua recomendação diária é elevada em situações como infecções, gravidez e amamentação, e em tabagistas. A dieta deficiente em vitamina C pode desencadear o escorbuto, doença caracterizada por manifestações hemorrágicas (petéquias, equimoses, sangramento das gengivas), edema nas articulações, fadiga, tonteiras, anorexia, alterações cutâneas, infecções e morte. ${ }^{24}$

Embora os dois nutrientes tenham se apresentado em quantidades médias adequadas para as necessidades nutricionais dos dois modelos construídos, não se pode afirmar que isso acontecerá em todas as construções de cardápios pelos usuários da dieta, visto que não existem listas de substituição que orientem o paciente. Da mesma forma, não é um planejamento individualizado, o que poderá levar à baixa ou alta ingestão dos nutrientes, dependendo das necessidades nutricionais de quem seguirá esta dieta. Somente uma dieta individualizada e acompanhada por profissional habilitado poderá afirmar um consumo dietético correto de todos os macronutrientes e micronutrientes.

Já em relação ao cálcio, o mesmo se apresentou com oferta baixa, em relação às necessidades nutricionais do modelo 2. Sua importância em quantidades adequadas é primordial para manutenção da saúde. Esse micronutriente participa de funções biológicas, como a contração muscular, mitose, coagulação sanguínea, transmissão do impulso nervoso ou sináptico e o suporte estrutural do esqueleto. Estudos têm evidenciado um consumo de cálcio abaixo do recomendado 
pela população brasileira e a associação entre o baixo consumo de cálcio e o desenvolvimento de DCNT, entre elas, osteoporose, câncer de cólon, hipertensão arterial e obesidade. ${ }^{23}$ Em dietas hipoglicídicas, a absorção de cálcio é diminuída, uma vez que o carboidrato parece aumentar a absorção desse micronutriente, principalmente a lactose..$^{25}$

Em relação a dietas hiperlipídicas, Buzinaro et al ${ }^{25}$ demonstraram que o acréscimo de gordura pode promover má absorção, ou esteatorreia, diminuindo assim a absorção do cálcio. Normalmente, isso é resultante da precipitação do cálcio com ácidos graxos devido à presença de sabões insolúveis no lúmen do intestino. O volume de sabões produzidos, paralelamente, é significativo para ionizar os ácidos de gordura, ao mesmo período em que diminui a concentração de cálcio. Em dietas hiperproteicas, esses mesmos autores defendem uma combinação entre aumento da ingestão proteica e piora do metabolismo do cálcio, sendo que tal interação se refere à ampliação na excreção de cálcio na urina..$^{25}$

As fibras encontram-se inadequadas nos dois modelos. Estas são polissacarídeos e polímeros de fenóis (lignina) presentes em alimentos como cereais, grãos, raízes e folhas. Apesar de serem resistentes à ação das enzimas digestivas humanas, podem ser parcialmente digeridas por bactérias do cólon ou eliminadas de maneira inalterada nas fezes. São também considerados alimentos funcionais, que auxiliam na melhoria das atividades vitais, proporcionam um bom desempenho do trato gastrointestinal, além de seu consumo adequado está relacionado a menores prevalências de doenças arteriais coronarianas (DAC), acidente vascular cerebral (AVC) e doença vascular periférica. A deficiência de fibras na dieta favorece o aumento dos distúrbios e doenças gastrointestinais, como a síndrome do cólon irritável, apendicite, diverticulite, doença de Crohn, hemorroidas e constipação intestinal ${ }^{26,27} \mathrm{O}$ consumo insuficiente de fibras nas dietas hiperproteica e hiperlipídica de ambos os modelos é possivelmente explicado pelo baixo consumo de frutas, vegetais e cereais integrais, devido à restrição de carboidratos, que é característica de tal dieta. ${ }^{27}$

Resultado preocupante também encontrado neste estudo foi o de oferta de colesterol dietético. O mesmo encontra-se oito vezes maior no caso do modelo $1 \mathrm{e}$, aproximadamente, 13 vezes maior em relação ao modelo 2, de acordo com a quantidade máxima recomendada para ambos os modelos. De toda forma, era esperado este resultado, uma vez que estas dietas têm como principal característica o alto consumo de proteínas e lipídeos e os alimentos permitidos livremente na mesma são aqueles de origem animal, principais fontes do colesterol. O colesterol é encontrado apenas em gorduras de origem animal, quase que inteiramente na forma livre. Suas principais fontes alimentares são a gema de ovo, leite e derivados, carne bovina, pele de aves e miúdos. Seu equilíbrio é resultante do balanço entre ingestão, absorção/excreção e síntese..$^{28,29}$

Segundo Lottenberg, ${ }^{28}$ desde a década de 1950 sabe-se que a gordura alimentar amplia o risco cardiovascular e que o consumo de gordura superior a $30 \%$ das calorias associa-se à maior incidência de aterosclerose. Na mesma época, determinou-se a forte relação entre obesidade, diabetes e 
inflamação, evidenciando, posteriormente, que metabolismo e imunidade são circunstâncias interdependentes e que excesso de ingestão alimentar e alimentos fontes de gordura e de colesterol induzem a um maior risco para DCNT.

Estudos demonstraram que intervenções alimentares corretas podem atenuar ou prevenir significativamente o aparecimento de diversas DCNT, tendo em vista que dietas ricas em colesterol e pobre em fibras elevam a colesterolemia, acarretam a aterosclerose precoce, hipertensão arterial, diabetes e obesidade. Além disso, concentrações elevadas de LDL aumentam o risco cardiovascular, ${ }^{29,30}$ o que é evidenciado na dieta avaliada no presente estudo.

A utilização de dietas inadequadas, sem o correto acompanhamento de profissional habilitado, auxilia no estabelecimento de elementos comuns, como a obesidade, diabetes e doenças cardiovasculares que acompanham o processo de Transição Nutricional e Transição Epidemiológica, em torno do qual oscilam outros distúrbios agregados, como as doenças osteoarticulares, as dislipidemias, a elevação dos níveis sanguíneos de ácido úrico e outras manifestações de caráter degenerativo. São estes elementos que predominam em populações com maior expectativa de vida, com uma dieta rica em lipídeos (particularmente, os de origem animal), açúcares e alimentos refinados, além de uma reduzida ingestão de carboidratos complexos e fibras, o que frequentemente se denomina como "dieta ocidental" ${ }^{31}$ Paralelamente, também acontecem alterações na composição corporal promovidas tanto pelo predomínio dessa dieta quanto pelo fato de está relacionada ao declínio progressivo da atividade física dos indivíduos, ambos proporcionando um aumento na prevalência da DCNT e de piora da qualidade de vida da população. ${ }^{32}$

\section{Conclusão}

Este estudo encontrou um planejamento alimentar inadequado para os modelos em sobrepeso construídos, possíveis usuários da "dieta da proteína" veiculada em mídia digital e impressa. Os resultados apresentam uma dieta hiperproteica, hiperlipídica, hipoglicídica e hipercalórica, além de valores acima do recomendado para o nutriente colesterol e abaixo do recomendado para os nutrientes cálcio e fibras, o que pode comprometer a saúde dos usuários da dieta em curto, médio e longo prazo.

Portanto, conclui-se que a dieta da proteína não pode ser considerada saudável e equilibrada, visto que não considera as necessidades nutricionais de indivíduos saudáveis ou indivíduos nutricionalmente vulneráveis, como aqueles que possuem predisposição a doenças cardiovasculares, uma vez que tal dieta não é realizada de forma individualizada.

A composição ideal de uma dieta é aquela que promove maior saciedade com ingestão energética adequada a cada fase da vida. Tanto para indivíduos saudáveis quanto para os obesos, os macronutrientes e micronutrientes devem estar em proporções equilibradas em relação ao Gasto 
Energético Total (GET). É importante também que a dieta seja composta por uma variedade de alimentos, apropriada aos hábitos ou padrões alimentares do individuo.

Dessa forma, é de extrema importância que sejam feitas campanhas de educação nutricional para a população em geral, esclarecendo os malefícios que uma dieta desbalanceada e sem acompanhamento nutricional pode gerar ao estado nutricional e a importância do atendimento e acompanhamento do paciente pelo profissional habilitado, o nutricionista.

\section{Colaboradores}

Faria, LN e Souza AA participaram da concepção, desenho do estudo, escrita, análise, interpretação e revisão do artigo.

Conflito de Interesses: Os autores declaram não haver conflito de interesses.

\section{Referências}

1. Porto ACV, Pires BAB, Coelho SC. Frequência de consumo de fast food em crianças de uma escola pública e uma escola privada do município de Nova Iguaçu no Rio de Janeiro e sua influência no perfil nutricional. Acta Pediatr Port. 2014; 44(6):301-5.

2. Soares LR, Pereira MLC, Mota MA, Jacob TA, Nakaoka VYES, Kashiwabara TGB. A transição da desnutrição para a obesidade. Braz J Surg Clin Res. 2013; 5(1):64-68.

3. Tardido AP, Falcão MA. O impacto da modernização na transição nutricional e na obesidade. Rev Bras Nutr Clin. 2006; 21(2):117-24.

4. Farias SJSS, Fortes RC, Fazzio DMG. Análise da composição nutricional de dietas da moda divulgadas por revistas não científicas. Nutrire 2014; 39(2):196-202.

5. Betoni F, Zanardo VPS, Ceni GC. Avaliação de utilização de dietas da moda por pacientes de um ambulatório de especialidades em nutrição e suas implicações no metabolismo. ConScientiae Saúde 2010; 9(3):430-440.

6. Atkins RC. A nova dieta revolucionária do Dr. Atkins. 14. ed. Rio de Janeiro: Records; 20044. 29 p.

7. Virtual Nutri Plus. 2012. [acesso em: 31 mar. 2016]. Disponível em: http://www.virtualnutriplus.com.br

8. Microsoft Project for Windows. Software Microsoft Office Excel, versão 2010. Microsoft Corporation; 2010.

9. World Health Organization. Distribuição percentual de macronutrients. Geneva: WHO; 2003.

10. Institute of Medicine. Dietary reference intake: applications in dietary assessment. food and nutrition board. Washington, DC: National Academy Press; 2001. 
11. Institute of Medicine. Dietary reference intake for energy, carbohydrate, fiber, fat, fatty acids, cholesterol, protein and amino acids. food and nutrition board. Washington, DC: National Academy Press; 2002.

12. Padovani RM, Amaya-Farfan J, Colugnati FAB, Domene SMA. Dietary reference intakes: aplicabilidade das tabelas em estudos nutricionais. Rev Nutr. 2006; 19(6):741-760.

13. World Healft Organization. Energy and protein requirements. Geneva: WHO, FAO; 1985. WHO Techinical Report Series, 724.

14. American College of Sports Medicine. ACSM's guidelines for exercise testing and prescription. Baltimore, MA; Lippincott Williams \& Wilkins; 2000.

15. Stringhini MLF, Costa e Silva JM, Oliveira FG. Vantagens e desvantagens da dieta Atkins no tratamento da obesidade. Salusvita 2007; 26(2):257-268.

16. Borba AJ. Efeito da dieta hiperlipídico-protéica no metabolismo de ratos Wistar adultos [dissertação]. [Uberaba]: Universidade Federal do Triângulo Mineiro; 2008.

17. Foster GD, Wyatt HR, Hill JO, Brian GM, Carrie BBS, Selma M, et al. A randomized trial of a low carbohydrate diet for obesity. N Engl J Med. 2003; 348:2082-2090.

18. Cipriano DO, Lima HV, Souza AAS, Silva MCFC, Santos ACO. Frequência de indivíduos com intolerância a glicose em jejum em um hospital universitário: comparação de critérios diagnósticos. J Bras Patol Med Lab. 2006; 42(6):419-423.

19. Weigle DS, Breen PA, Matthys CC, Callahan HS, Meeuws KE, Burden VR, et al. A high-protein diet induces sustained reductions in appetite, ad libitum caloric intake, and body weight despite compensatory changes in diurnal plasma leptin and ghrelin concentrations. Am J Clin Nutr. 2005; 82(1):41-48.

20. Pedrosa RG, Donato Junior J, Tirapegui J. Dieta rica em proteína na redução do peso corporal. Rev Nutr. 2009; 22(1):105-111.

21. Di Vetta V, Clarisse M, Giusti V. Hypocaloric diets: which ones to adise/avoid? Rev Med Suisse 2005; 1(12):818-22.

22. Garcês BP, Pereira ES, Sousa OS, Sousa JP, Valadares MC. A importância do ferro para a vida humana. $53^{\circ}$ Congresso Brasileiro de Química; 14-18 out. 2013; Rio de Janeiro.

23. Pereira, Giselle AP, Genaro PS, Pinheiro MM, Sezjnfeld VL, Martini LA. Cálcio dietético: estratégias para otimizar o consumo. Rev Bras Reumatol. 2009; 49(2):164-171.

24. Manela-Azulay M. Vitamina C. An Bras Dermatol. 2003; 78(3):265-272.

25. Buzinaro EF, Almeida RNA de, Mazeto GMFS. Biodisponibilidade do cálcio dietético. Arq Bras Endocrinol Metab. 2006; 50(5):852-861.

26. Bernaud FSR, Rodrigues TC. Fibra alimentar: ingestão adequada e efeitos sobre a saúde do metabolismo. Arq Bras Endocrinol Metab. 2013; 57(6):397-405.

27. Reis NT. Nutrição clínica: sistema digestório. Rio de Janeiro: Rubio; 2003. 294 p. 
28. Lottenberg AMP. Importância da gordura alimentar na prevenção e no controle de distúrbios metabólicos e da doença cardiovascular. Arq Bras Endocrinol Metab. 2009; 53(5):595-607.

29. Patel MD, Thompson PD. Phytosterols and vascular disease. Atherosclerosis 2006; 186(1):12-9.

30. Howard BV, Van Horn L, Hsia J, Manson JE, Stefanick ML, Wassertheil-Smoller S. Low-fat dietary pattern and risk of cardiovascular disease: the women's health initiative randomized controlled dietary modification trial. JAMA 2006; 295(6):655-66.

31. De Lorgeril M, Salen P. The mediterranean-style diet for the prevention of cardiovascular diseases. Public Health Nutr. 2006; 9(1A):118-123.

32. Lima LP, Sampaio HAC. Caracterização socioeconômica, antropométrica e alimentar de obesos graves. Ciênc Saúde Coletiva 2007; 12(4):1011-1020.

Recebido: 28/07/2016

Revisado: 05/02/2017

Aceito: 15/05/2017 